\title{
Relationship between maternal endocrine environment, early embryo development and inhibition of the luteolytic mechanism in cows
}

\author{
G. E. Mann and G. E. Lamming \\ University of Nottingham, School of Biosciences, Division of Animal Physiology, \\ Sutton Bonington, Loughborough LE12 5RD, UK
}

In this study, the relationship between maternal hormone environment and early embryo development in mature non-lactating Holstein-Friesian cows was investigated. Animals were inseminated at either 72 or $96 \mathrm{~h}$ after prostaglandin injection $(n=23)$ or were left as uninseminated controls $(n=10)$. Plasma samples were collected once a day from the first day of insemination (day 1) until day 16, when the cows underwent an oxytocin challenge, and were then slaughtered and their reproductive tracts removed. The tracts were flushed to collect embryos and the flushes were measured for interferon tau (IFN- $\tau$ ) activity. Inseminated cows without an embryo on day $16(n=5)$ underwent both delayed ovulation (indicated by delayed decrease in oestradiol concentrations) and a delayed increase in progesterone concentrations after ovulation compared with cows with an embryo on day $16(n=15)$. Within the group of cows with an embryo, those with poorly developed embryos producing undetectable concentrations of IFN- $\tau(n=7)$ had similar

\section{Introduction}

During early pregnancy, embryos must inhibit the development of the luteolytic mechanism to maintain the secretion of progesterone necessary for continuing development. Thus, embryos must secrete a protein, interferon tau (IFN$\tau)$, which acts locally within the uterus to inhibit luteolytic $\mathrm{PFG}_{2 \alpha}$ secretion by inhibiting the development of oxytocin receptors on the luminal epithelium (Robinson et al., 1999) and through the induction of a prostaglandin synthesis inhibitor (Thatcher et al., 1995). A full understanding of the control of embryo development and IFN- $\tau$ production is of paramount importance in determining strategies to reduce the high early embryo mortality that occurs in dairy cattle.

The principal hormone implicated in the control of this process is progesterone. Progesterone plays a major role in stimulating the production of a variety of endometrial secretions necessary for the successful development of embryos (for review see Geisert et al., 1992). Furthermore, a number

Email: george.mann@nottingham.ac.uk oestradiol profiles but underwent a delayed progesterone increase after ovulation compared with cows with well developed embryos producing measurable quantities of IFN- $\tau$ $(n=8)$. In the cows with an embryo, the plasma concentration of 13,14-dihydro-15-keto $\mathrm{PGF}_{2 \alpha,}$ the principal metabolite of $\mathrm{PGF}_{2 \alpha \prime}$ after injection of oxytocin was lower than that of control cows and cows without an embryo. However, when the cows with an embryo were compared on the basis of production of embryonic IFN- $\tau$, the PGF $_{2 \alpha}$ response to oxytocin was attenuated completely in cows that had measurable IFN- $\tau$ activity, whereas a response of similar magnitude to that in control cows and cows without an embryo was observed in those with undetectable IFN- $\tau$ activities. In conclusion, the successful maternal recognition of pregnancy in cows depends on the presence of a sufficiently well developed embryo producing sufficient quantities of IFN- $\tau$, which is, in turn, dependent on an appropriate pattern of maternal progesterone secretion.

of studies have revealed lower maternal progesterone concentrations in both the milk and plasma of inseminated cows in which pregnancy fails compared with cows in which pregnancy is established successfully (Henricks et al., 1971; Lukaszewska and Hansel, 1980; Lamming et al., 1989; Mann et al., 1995; Butler et al., 1996). Studies in sheep have shown that low progesterone concentrations can lead to poor embryo development (Nephew et al., 1991) and, in cows, supplementary progesterone enhances the development of conceptuses (Garrett et al., 1988).

In the present study, IFN- $\tau$ production by day 16 bovine embryos was measured and IFN- $\tau$ activities were compared with maternal endocrine profiles to identify hormonal patterns conducive to good embryonic development. Embryonic development was then related to the efficiency of inhibition of the luteolytic mechanism.

\section{Materials and Methods}

\section{Experimental animals}

The study was performed in 33 mature non-lactating Holstein-Friesian cows. All cows had calved at least once 
and were obtained at the end of lactation from the University of Nottingham commercial herd. The cows were maintained on a diet of hay and concentrate pellets throughout the study. The study was carried out with Home Office approval.

The oestrous cycles of the cows were synchronized by two injections of the $\mathrm{PGF}_{2 \alpha}$ analogue, cloprostenol (Estrumate; Schering-Plough Animal Health, Welwyn Garden City) administered at an 11-13 day interval. Cows were inseminated at either 72 or $96 \mathrm{~h}$ after the second injection of cloprostenol $(n=23)$ by Genus technicians (Genus Plc, Westmere Drive, Crewe) using semen from beef bulls, or were kept as uninseminated controls $(n=10)$.

\section{Blood sampling and oxytocin challenges}

Before the second injection of cloprostenol, a jugular vein of each animal was cannulated under local anaesthesia $(2 \mathrm{ml}$ lignocaine s.c. as Lignovet $2 \%(\mathrm{w} / \mathrm{v})$ C-Vet, Bury St Edmonds) with a $30 \mathrm{~cm}$ indwelling catheter (Secalon universal tubing; BOC Health Care, Swindon) using a 12 gauge needle and guide wire. The cannulae were maintained for the duration of the experiment and were used for the collection of blood samples. Plasma samples for determination of progesterone and oestradiol concentrations were collected daily at 09:00 h, starting on the first day of insemination (defined as day 1 ). All samples were collected into heparinized tubes, centrifuged at $1500 \mathrm{~g}$ for $10 \mathrm{~min}$ and the plasma samples were stored at $-20^{\circ} \mathrm{C}$.

Plasma samples were collected at 20 min intervals for $8 \mathrm{~h}$ on days 14 and 15 to determine plasma concentrations of 13,14-dihydro-15-keto $\mathrm{PGF}_{2 \alpha}$ (PGFM), the principal metabolite of $\mathrm{PGF}_{2 \alpha}$, to monitor the development of the luteolytic mechanism. On day 16 after first insemination, plasma samples were collected at 20 min intervals for $4 \mathrm{~h}$. A single i.v. bolus of $50 \mathrm{iu}$ oxytocin (Hoechst UK Ltd, Walton Manor, Milton Keynes) in $5 \mathrm{ml}$ saline was then administered to the cows, followed by flushing with a further $5 \mathrm{ml}$ saline and plasma samples were collected at $10 \mathrm{~min}$ intervals for a further $1 \mathrm{~h}$. All samples were collected into heparinized tubes, centrifuged at $1500 \mathrm{~g}$ for $10 \mathrm{~min}$ and the plasma samples were stored at $-20^{\circ} \mathrm{C}$.

\section{Embryo collection}

Immediately after collection of the final blood sample on day 16 the cows were transported to an adjacent abattoir and the uterus of each cow was collected immediately after death (by captive bolt and exsanguination) and transported to the laboratory. The uterine horns were dissected free from the surrounding tissues and separated past the bifurcation. Each horn was flushed with $20 \mathrm{ml}$ modified Eagle's medium (Gibco Ltd, Paisley) into a single dish and the embryo, if present, was classified as spherical $(<0.5$ $\mathrm{cm})$, tubular $(0.5-1.0 \mathrm{~cm})$ or filamentous $(>1 \mathrm{~cm})$. The uterine flushings were frozen at $-20^{\circ} \mathrm{C}$ until subsequently analysed for IFN $-\tau$ content by antiviral assay.

\section{Hormone assays}

Progesterone concentrations were measured in plasma samples by radioimmunoassay as described by Haresign et al. (1975). The intra- and interassay coefficients of variation were 11.2 and $12.6 \%$, respectively, and the sensitivity of the assay was $0.5 \mathrm{ng} \mathrm{m}^{-1}$.

Oestradiol concentrations were measured in plasma samples after extraction with diethyl ether using an assay kit (E2 MAIA; Serono Diagnostics Ltd, Woking) modified for use in cows (Mann et al., 1995). The intra- and interassay coefficients of variation were 5.9 and $9.3 \%$, respectively, and the sensitivity of the assay was $0.5 \mathrm{pg} \mathrm{ml}^{-1}$.

Radioimmunoassay was used to determine PGFM concentrations in plasma samples after extraction with acidified diethyl ether (Kaker et al., 1984). The sensitivity of the assay was $30 \mathrm{pg} \mathrm{ml}^{-1}$, and the intra- and interassay coefficients of variation were 13.5 and $15.6 \%$, respectively.

The IFN- $\tau$ activity of samples was measured by MDBK cell Semiliki Forest virus cytoplasmic inhibition assay (Abayasekara et al., 1995). All samples with IFN- $\tau$ activity below the initial detection limit of 100 anti-viral units (avu) $\mathrm{ml}^{-1}$ were concentrated by 10 times by centrifugal filtration using Microceps ${ }^{\mathrm{TM}}$ (Flowgen Instruments Ltd, Lichfield) with a molecular cut off of $3 \mathrm{~K}$. A $3 \mathrm{ml}$ aliquot of flushing medium was used and was reconstituted in $300 \mu \mathrm{l}$ medium. Of nine samples in which measurement of IFN- $\tau$ activity was not initially possible, measurements were obtained from two flushes after concentration of the samples, leaving seven flushes in which measurements were still not possible. Five samples in which IFN- $\tau$ activity was measured successfully in the assay were also concentrated by 10 times to validate this approach, resulting in a mean \pm SEM increase in IFN- $\tau$ activity of $913 \pm 58 \%$. After concentration of the samples, the limit of detection was 10 avu $\mathrm{ml}^{-1}$, which is equivalent to $4 \times 10^{2}$ avu $40 \mathrm{ml}$ per uterine flush.

\section{Statistical analysis}

Statistical analysis of changes in hormone concentrations within groups was performed using repeated sample ANOVA. Significant episodes of release of $\mathrm{PGF}_{2 \alpha}$ were defined to have occurred when the plasma concentration of PGFM in at least two consecutive samples taken at $1 \mathrm{~h}$ intervals was $>$ baseline +2 SD. Differences in the proportion of cows undergoing such episodes were compared by chi-squared test. Significant responses to oxytocin were defined to have occurred when the concentration of PGFM at three consecutive time points after the bolus challenge was greater than the pre-treatment mean $+2 \mathrm{SD}$ and were defined to have ended when the concentration in a single sample decreased to below this concentration. Differences in the magnitude of responses to oxytocin were analysed by ANOVA and differences in the proportion of cows responding were analysed by chi-squared test. 


\section{Results}

\section{Pregnancy status}

One control cow and three inseminated cows had become cystic by the time they were killed and were excluded from further analysis, leaving a total of nine control cows and 20 inseminated cows. Embryos were recovered from 15 inseminated cows, whereas no embryo was found in the other five cows. For the purposes of analysis, cows were divided initially into three groups: control $(n=9)$, embryo positive $(n=15)$ and embryo negative $(n=5)$. Embryo positive cows were further subdivided into those with detectable IFN- $\tau$ activity in uterine flushes (IFN positive: $>4 \times 10^{2}$ avu per flush; $n=8$ ) and those with undetectable IFN- $\tau$ activity (IFN negative: $<4 \times 10^{2}$ avu per flush; $n=7$ ).

\section{Plasma concentrations of progesterone and oestradiol}

Plasma oestradiol concentrations were significantly higher in the embryo negative group than in either the control $(P<0.01)$ or embryo positive $(P<0.001)$ group on day 1 (first day of insemination) and were also significantly higher than in the embryo positive group on days 2 $(P<0.01)$ and $3(P<0.05)$ (Fig. 1$)$. The increased plasma oestradiol concentrations observed on days 1 and 2 are indicative of delayed ovulation. After day 3, there were no significant differences in plasma oestradiol concentrations among the three groups.

Cows in the embryo negative group underwent a significantly delayed increase (to $>1 \mathrm{ng} \mathrm{ml}^{-1}$ ) in progesterone concentrations after ovulation (day $6.2 \pm 0.4$ ) compared with cows in the embryo positive (day $4.9 \pm 0.2$ ) and control (day $5.0 \pm 0.3$ ) groups $(P<0.01)$ (Fig. 1). As well as a delayed increase in progesterone concentration, the overall concentration of progesterone during the luteal phase was lower in the embryo negative group than in the embryo positive group $(P<0.05)$.

\section{Embryo development and production of IFN- $\tau$}

IFN- $\tau$ was detected in the uterine flushings of eight of 15 cows (mean $9.6 \times 10^{3} \pm 2.9 \times 10^{3}$ avu per flush; range $7.2 \times 10^{2}-2.4 \times 10^{4}$ avu per flush) from which an embryo was recovered (IFN positive). IFN- $\tau$ concentrations were below the detection limit of the assay in the other seven cows (IFN negative). Embryos were still at the spherical or tubular stages $(<1 \mathrm{~cm}$ in length) in the IFN negative group, whereas embryos from cows in the IFN positive group had developed to the tubular or filamentous stages of development (>0.5 cm in length). Cows in the IFN negative group underwent a delayed increase in progesterone concentrations after ovulation ( $1 \mathrm{ng} \mathrm{ml}^{-1}$ on day $5.6 \pm 0.4$ ) compared with IFN positive cows (day $4.1 \pm 0.1$; $P<0.01)$ and had a lower final luteal phase 'plateau' between days 12 and $16 \quad\left(6.1 \pm 0.4 \mathrm{ng} \mathrm{ml}^{-1}\right.$ versus $7.8 \pm 0.8 \mathrm{ng} \mathrm{ml}^{-1} ; P<0.05$ ) (Fig. 2). In contrast to progesterone, there were no significant differences in plasma

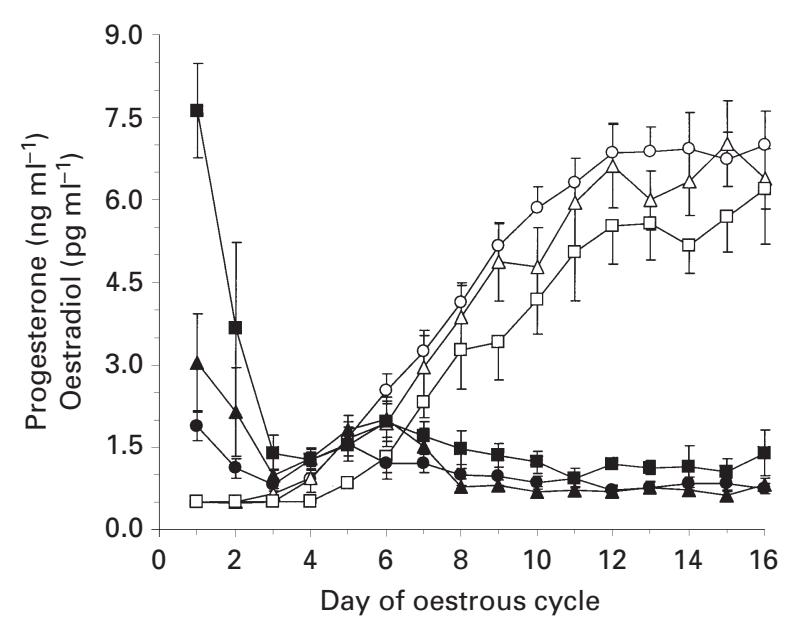

Fig. 1. Plasma oestradiol concentrations in uninseminated cows $(\boldsymbol{\Lambda}$, control; $n=9)$ and inseminated cows with $(\boldsymbol{\bullet}$, embryo positive; $n=15$ ) and without ( $\boldsymbol{\square}$, embryo negative; $n=5$ ) an embryo on day 16 after first insemination, and plasma progesterone concentrations in uninseminated cows $(\triangle$, control; $n=9)$ and inseminated cows with $(\bigcirc$, embryo positive; $n=15)$ and without $(\square$, embryo negative; $n=5$ ) an embryo on day 16 . Values are mean \pm SEM.

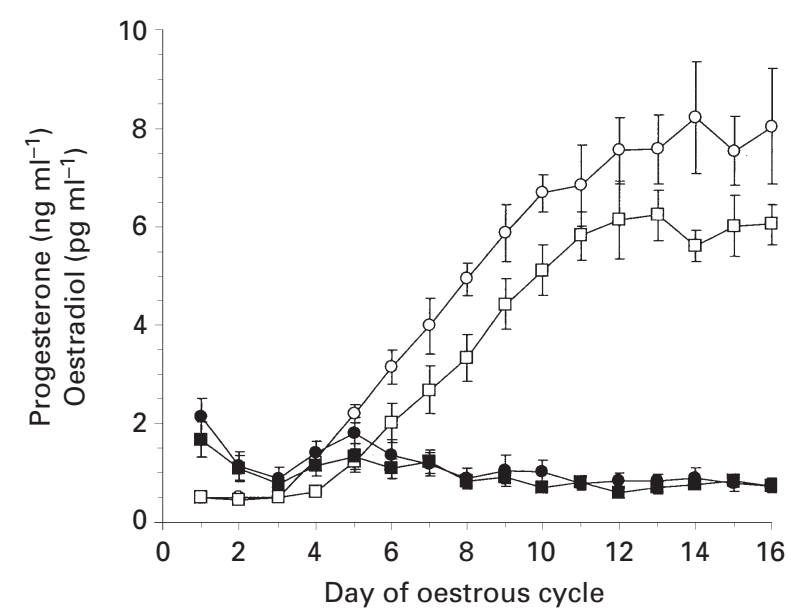

Fig. 2. Plasma progesterone concentrations in inseminated cows with embryos that had measurable $(\bigcirc$, IFN positive; $n=8)$ or unmeasurable ( $\square$, IFN negative; $n=7$ ) interferon tau activity on day 16 after first insemination, and plasma oestradiol concentrations in inseminated cows with embryos that had measurable $(\boldsymbol{O}$, IFN positive; $n=8$ ) or unmeasurable ( $\boldsymbol{\square}$, IFN negative; $n=7$ ) interferon tau activity on day 16 after first insemination. Values are mean \pm SEM.

oestradiol concentrations between cows in the IFN positive and IFN negative groups (Fig. 2).

\section{Plasma concentrations of PGFM}

Mean plasma concentrations of PGFM during the sampling periods on days 14 and 15, and before the oxytocin challenge on day 16, did not differ between the control, 
Table 1. Plasma 13,14-dihydro-15-keto $\mathrm{PGF}_{2 \alpha}$ (PGFM) concentrations in samples from cows collected at $1 \mathrm{~h}$ intervals for $8 \mathrm{~h}$ on days 14 and 15 , and for $4 \mathrm{~h}$ on day 16 after first insemination

\begin{tabular}{lccc}
\hline & \multicolumn{3}{c}{ PGFM $\left(\mathrm{pg} \mathrm{m} \mathrm{m}^{-1}\right)$} \\
\cline { 2 - 4 } Animals & Day 14 & Day 15 & Day 16 \\
\hline Control $(n=9)$ & $40.3 \pm 2.4$ & $38.9 \pm 1.9$ & $41.5 \pm 1.7$ \\
Embryo negative $(n=5)$ & $42.7 \pm 4.9$ & $40.5 \pm 4.4$ & $40.8 \pm 4.4$ \\
Embryo positive $(n=15)$ & $39.8 \pm 1.4$ & $38.4 \pm 1.3$ & $39.3 \pm 1.6$ \\
IFN positive $(n=8)$ & $41.7 \pm 2.7$ & $39.1 \pm 2.4$ & $40.1 \pm 3.1$ \\
IFN negative $(n=7)$ & $38.1 \pm 0.8$ & $37.7 \pm 1.5$ & $38.5 \pm 1.4$ \\
\hline
\end{tabular}

Values are mean \pm SEM.

Embryo negative: cow without embryo; embryo positive: cow with embryo; IFN positive: cows with detectable interferon tau activity in uterine flushes; IFN negative: cows with undetectable interferon tau activity in uterine flushes.

embryo positive and embryo negative groups, or among days (Table 1). Furthermore, in the embryo positive group there was no differences between the IFN positive and IFN negative cows. No endogenous PGFM episodes were detected in any animals on day 14 . On day 15, episodes were detected in two of five embryo negative and two of nine control cows compared with none of 15 embryo positive cows $(P<0.05)$.

Cows in the control and embryo negative groups underwent similar consistent increases in mean plasma PGFM concentrations in response to oxytocin, whereas this response was significantly $(P<0.01)$ attenuated in cows in the embryo positive group (Fig. 3). In the control and embryo negative groups, 13 of 14 cows underwent a significant response to oxytocin compared with seven of 15 cows in the embryo positive group $(P<0.01)$. Within the embryo positive group, the mean plasma concentrations of PGFM after oxytocin challenge in the IFN positive cows $\left(43.0 \pm 3.2 \mathrm{pg} \mathrm{ml}^{-1}\right)$ were significantly $(P<0.01)$ lower than in the IFN negative cows $\left(62.8 \pm 5.5 \mathrm{pg} \mathrm{ml}^{-1}\right)$ (Fig. 4). However, the mean PGFM concentrations in IFN negative cows were not significantly different from those of cows in the control $\left(74.0 \pm 8.6 \mathrm{pg} \mathrm{ml}^{-1}\right)$ or embryo negative $(74.6$ $\pm 8.4 \mathrm{pg} \mathrm{ml}^{-1}$ ) groups.

\section{Discussion}

The importance of progesterone during early pregnancy in cows is well established. Numerous studies have demonstrated a relationship between low maternal progesterone concentrations and pregnancy failure, and further studies have demonstrated both improved pregnancy rates (for review see Mann and Lamming, 1999) and increased embryo development (Garrett et al., 1988) after progesterone supplementation. However, the precise relationship between maternal hormone environment and embryo development has not been established. In the present study, it is demonstrated that the degree of embryo development is closely related to the maternal progesterone environment. Cows with poorly developed embryos on day 16 (day 16 after first insemination) that were producing little

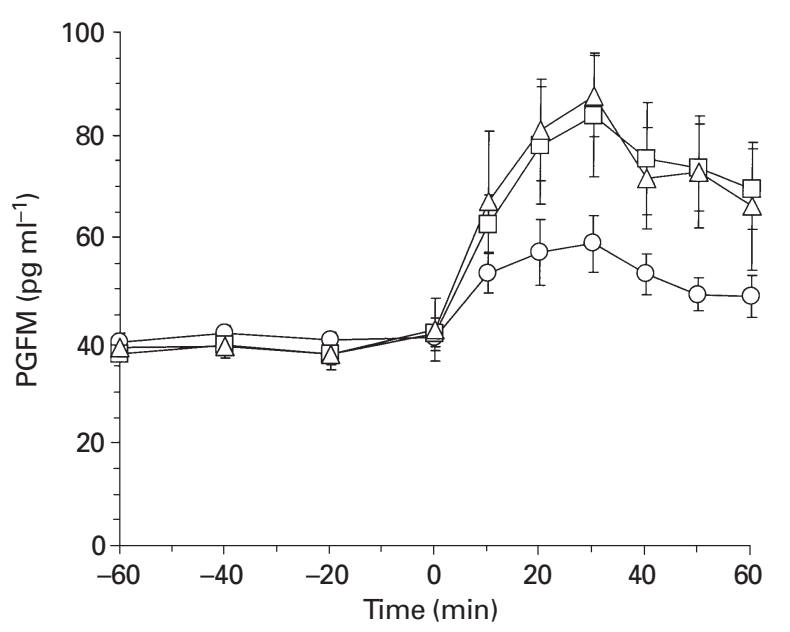

Fig. 3. Plasma 13,14-dihydro-15-keto $\mathrm{PGF}_{2 \alpha}$ (PGFM) concentrations before and after injection of 50 iu oxytocin on day 16 in uninseminated cows $(\triangle$, control; $n=9)$ and in inseminated cows with $(\bigcirc$, embryo positive; $n=15)$ and without $(\square$, embryo negative; $n=5$ ) an embryo on day 16 after first insemination. Values are mean \pm SEM.

or no IFN- $\tau$ exhibited both a delayed increase in progesterone concentrations after ovulation and had a lower luteal phase plateau than did cows with well developed embryos.

Day 16 was chosen as the day of slaughter of the cows in the present study as the aim was to investigate the hormonal factors leading to poor embryo development and not the factors resulting from a failure of the embryo to prevent initiation of luteolysis. As soon as progesterone concentrations start to decrease at the onset of luteal regression, oestradiol secretion increases and a cascade of reproductive processes is triggered, making it impossible to relate physiological comparisons to embryo development. In the present study, no significant decrease in progesterone concentrations or increase in oestradiol concentrations was apparent at the end of the sampling period. However, in a previous study on cows from the 


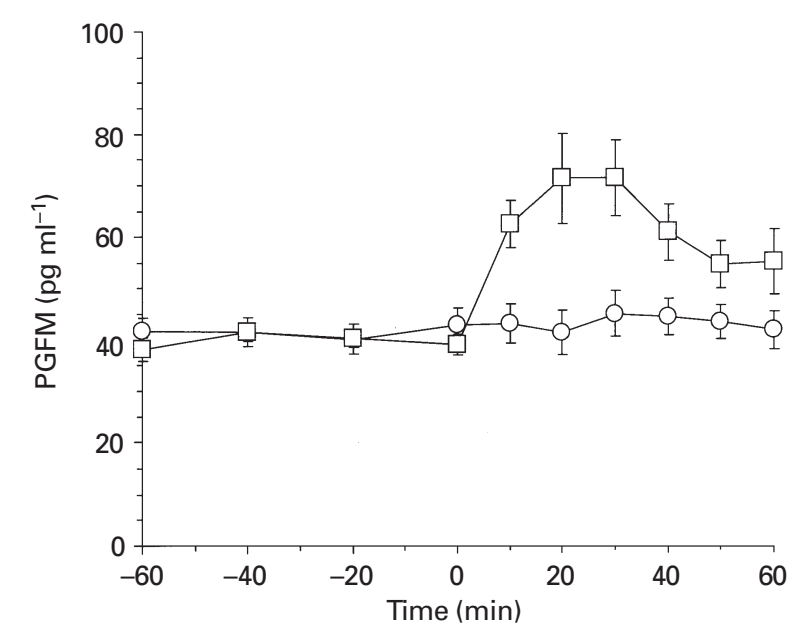

Fig. 4. Plasma 13,14-dihydro-15-keto $\mathrm{PGF}_{2 \alpha}$ (PGFM) before and after injection of 50 iu oxytocin on day 16 in inseminated cows with embryos that had measurable $(\bigcirc$, IFN positive; $n=8)$ or unmeasurable ( $\square$, IFN negative; $n=7$ ) interferon tau activity on day 16 after first insemination. Values are mean \pm SEM.

same source, it was found that by day 17 of the oestrous cycle mean progesterone concentrations had decreased, thus allowing oestradiol concentrations to increase (Mann et al., 1995).

Although the time of ovulation was not determined in the present study, increased plasma oestradiol concentrations measured in samples collected on day 1 (day of first artificial insemination), and in particular on day 2, 72 and $96 \mathrm{~h}$ after prostaglandin injection, were used as indicators of delayed ovulation. A number of studies in cows have demonstrated a marked decrease in oestradiol concentrations before ovulation (Chenault et al., 1975; Schams et al., 1977; Kaneko et al., 1991). Thus, an increased plasma concentration of oestradiol on any day indicates that ovulation has not yet occurred. Furthermore, it has been demonstrated that ovulation does not occur until about 12-24 h after preovulatory oestradiol concentrations have decreased to $<2 \mathrm{pg} \mathrm{ml}^{-1}$ (G. E. Mann, unpublished).

If this criterion is used, it can be seen that in the embryo negative group the lack of an embryo in the uterus on day 16 was associated with a marked delay in ovulation. It is likely that in these cows late ovulation may have resulted in failed fertilization. The marked delay in the increase in progesterone concentrations after ovulation observed in cows in the embryo negative group compared with those in the embryo positive and control groups can be accounted for by the delay in the timing of ovulation (indicated by a delayed decrease in oestradiol concentrations). However, the equally marked delay in the increase in progesterone concentrations after ovulation in the IFN negative group compared with the IFN positive group cannot be discounted in this way, as ovulation in these two groups appeared to have occurred at the same time. In this situation, the poor increase in progesterone concentrations after ovulation may have resulted from either poor luteinization or inadequate $\mathrm{LH}$ support of progesterone secretion (Garverick et al., 1992).

Although it is obviously impossible to determine the outcome of pregnancy in the cows that were killed on day 16 , it was apparent that a number of poorly developed embryos failed to inhibit oxytocin-induced $\mathrm{PGF}_{2 \alpha}$ release, whereas a number of well developed embryos did inhibit luteolytic $\mathrm{PGF}_{2 \alpha}$ release. This failure to inhibit luteolytic $\mathrm{PGF}_{2 \alpha}$ release was presumably due to the failure of poorly developed embryos to produce antiluteolytic IFN- $\tau$ in sufficient quantities. The mechanism by which adequate amounts of IFN- $\tau$ produced by well developed embryos inhibit luteolysis is now well established and involves inhibition of oxytocin receptors on the luminal epithelium (Robinson et al., 1999) and induction of a prostaglandin synthesis inhibitor (Thatcher et al., 1995).

It has been demonstrated previously that treatment with progesterone early in the luteal phase both advances the development of the embryo and increases production of IFN- $\tau$ (Garret et al., 1988). However, the present study is the first in which the relationship between endogenous progesterone secretion and the degree of embryo development and IFN- $\tau$ production have been quantified. As there is no evidence in cows that indicates a direct stimulatory effect of progesterone on the embryo, the link between progesterone and enhanced embryonic development is probably mediated through the effects of progesterone on the uterine environment.

In conclusion, the successful recognition of pregnancy in cows relies on an adequate degree of embryonic development and production of IFN- $\tau$, which is in turn dependent on an appropriate maternal hormone environment and, in particular, an appropriate pattern of progesterone secretion after ovulation.

The authors are grateful to S. J. Mann for sample collection and analysis, P. A. Fisher for carrying out the antiviral assays and the staff of the Nottingham University Animal Research Unit for care of the animals. The work was supported by the Milk Marketing Board of England and Wales and by MAFF and MDC under the Link Sustainable Livestock Production Programme. Hoechst donated the oxytocin and the PGFM antiserum was a gift from H. Dobson.

\section{References}

Abayasekara DRE, Sheldrick EL, Flicksmith HC and Flint APF (1995) Role of protein kinase $\mathrm{C}$ in the inhibitory action of trophoblast interferons on expression of the oxytocin receptor in sheep endometrium Endocrine 3 151-158

Butler WR, Calaman JJ and Beam SW (1996) Plasma and milk urea nitrogen in relation to pregnancy rate in lactating dairy cattle Journal of Animal Science 74 858-865

Chenault JR, Thatcher WW, Kalra PS, Abrams RM and Wilcox CJ (1975) Transitory changes in plasma progestins, estradiol and luteinising hormone approaching ovulation in the bovine Journal of Dairy Science $\mathbf{5 8}$ $709-717$

Garrett JE, Geisert RD, Zavy MT and Morgan GL (1988) Evidence for maternal regulation of early conceptus growth and development in beef cattle Journal of Reproduction and Fertility 84 437-446 
Garverick HA, Zollers WG and Smith MF (1992) Mechanisms associated with corpus luteum lifespan in animals having normal or subnormal luteal function Animal Reproduction Science 28 111-124

Geisert RD, Morgan GL, Short EC and Zavy MT (1992) Endocrine events associated with endometrial function and conceptus development in cattle Reproduction Fertility and Development 4 301-305

Haresign W, Foster JP, Haynes NB, Crighton DB and Lamming GE (1975) Progesterone levels following treatment of seasonally anoestrous ewes with synthetic LH-releasing hormone Journal of Reproduction and Fertility 43 269-279

Henricks DM, Lamond DR, Hill JR and Dickey JF (1971) Plasma progesterone concentrations before mating and in early pregnancy in the beef heifer Journal of Animal Science 33 450-454

Kaker ML, Murray RD and Dobson H (1984) Plasma hormone changes in cows during induced or spontaneous calvings and the early post partum period Veterinary Record 115 378-382

Kaneko H, Terads T, Watanabe G, Sasamoto S, Hasegawa $Y$ and Igarash M (1991) Ovulatory follicle dynamics and the concentration of oestradiol $17 \beta$, progesterone, luteinising hormone and follicle stimulating hormone during the periovulatory phase of the oestrous cycle in the cow Reproduction Fertility and Development 3 529-535

Lamming GE, Darwash AO and Back HL (1989) Corpus luteum function in dairy cows and embryo mortality Journal of Reproduction and Fertility Supplement 37 245-252

Lukaszewska J and Hansel W (1980) Corpus luteum maintenance during early pregnancy in the cow Journal of Reproduction and Fertility $\mathbf{5 9}$ 485-493
Mann GE and Lamming GE (1999) The influence of progesterone during early pregnancy in cattle Reproduction in Domestic Animals 34 269-274

Mann GE, Lamming GE and Fray MD (1995) Plasma oestradiol during early pregnancy in the cow and the effects of treatment with buserelin Animal Reproduction Science 37 121-131

Nephew KP, McClure KE, Ott T, Budois DH, Bazer FW and Pope WF (1991) Relationship between variation in conceptus development and differences in estrous cycle duration in ewes Biology of Reproduction 44 536-539

Robinson RS, Mann GE, Lamming GE and Wathes DC (1999) The effect of pregnancy on the expression of uterine oxytocin, oestrogen and progesterone receptors during early pregnancy in the cow Journal of Endocrinology $16021-33$

Schams D, Shallenberger E, Hoffmasnn B and Karg H (1977) The oestrous cycle of the cow: hormonal parameters and time relationships concerning oestrus, ovulation and electrical resistance of the vaginal mucus Acta Endocrinologica 86 180-192

Thatcher WW, Meyer MD and Danet-Desnoyers G (1995) Materna recognition of pregnancy Journal of Reproduction and Fertility Supplement 49 15-28

Received 12 June 2000

Accepted 23 August 2000. 